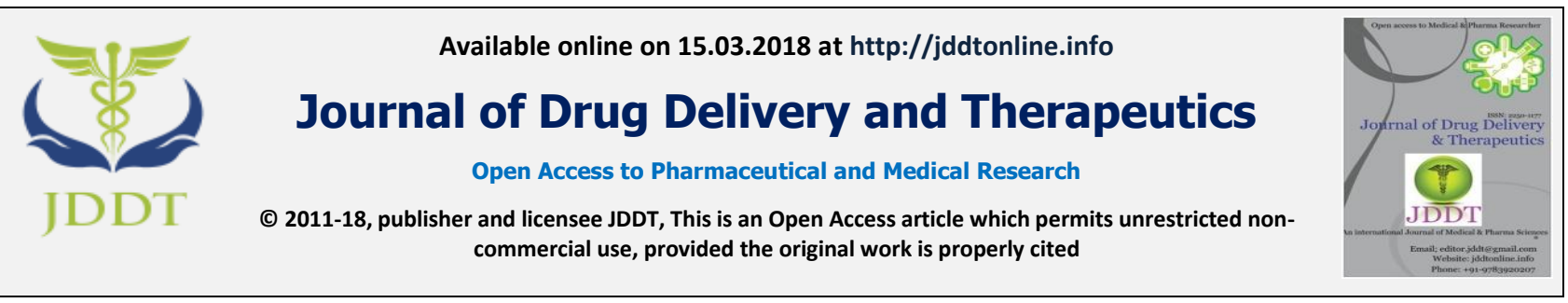

Open $\odot$ Access

Research Article

\title{
PREPARATION, CHARACTERIZATION AND EVALUATION OF NEBIVOLOL LOADED CHITOSAN NANOPARTICLES
}

\author{
Sharma Mayank ${ }^{1}$, Sharma Rajesh ${ }^{1 *}$, Jain Dinesh Kumar ${ }^{2}$ \\ ${ }^{1}$ School of Pharmacy, Devi Ahilya Vishwavidyalaya, Takshashila Campus, Khandwa Road, Indore-452001, India \\ ${ }^{2}$ College of Pharmacy, I.P.S Academy, Knowledge Village, Rajendra Nagar A.B. Road, Indore-452012, India
}

\begin{abstract}
Nebivolol (NEB) is an antihypertensive drug with poor oral bioavailability (12\%) in humans due to extensive first pass hepatic metabolism. Present work is an attempt to improve oral bioavailability of nebivolol by incorporating it with biodegradable polymer chitosan and preparing its nanoparticles. The results indicated stable nanoperticles with the value of $\mathrm{ZP}$ to be $+36.4 \mathrm{mV} \pm 2 \mathrm{mV}$, the small particle size of $79.23 \pm 45 \mathrm{~nm}$ and high entrapment efficiency of $72.56 \%$. The in vitro release study revealed sustained release of drug for $72 \mathrm{~h}$ with $71.24 \%$ cumulative drug release. The promising results from the study revealed the applicability of chitosan in the formulation of NEB loaded CNPs.
\end{abstract}

Keywords: Nebivolol, chitosan, ionic gelation technique, nanoparticles, oral bioavailability, poor water solubility

Article Info: Received 20 Jan, 2018; Review Completed 8 March, 2018; Accepted 9 March, 2018; Available online 15 March, 2018

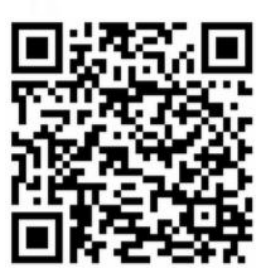

Cite this article as:

Sharma M, Sharma R, Jain DK, Preparation, characterization of nebivolol loaded chitosan nanoparticles ,

Journal of Drug Delivery and Therapeutics. 2018; 8(2):118-122

DOI: $\underline{\text { http://dx.doi.org/10.22270/jddt.v8i2.1730 }}$

*Address for Correspondence:

Sharma Rajesh, School of Pharmacy, Devi Ahilya Vishwavidyalaya, Takshashila Campus, Khandwa Road, Indore-452001, India

\section{INTRODUCTION}

High blood pressure is estimated to cause 7.1 million deaths, about 13 percent of the global fatality total. It is believed this number will grow to approximately 11 million by the year 2020 . Heart disease is the leading cause of death worldwide each year. Cardiovascular disease is also the leading cause of death in India, accounting for over 4 million deaths each year. Hypertension is the leading risk factor for cardiovascular and renal disease, increasing the risk of myocardial infarction, stroke, congestive heart failure, ruptured aortic aneurysm, and renal disease. It is clearly understandable that a more rational approach to diagnosing and treating high blood pressure could have a substantial impact on population morbidity and mortality. ${ }^{1}$

Oral administration of drugs is considered to be the most natural, uncomplicated, convenient and safe method.
Since nearly one third of drugs are poorly water soluble, oral bioavailability of those drugs could be issue. ${ }^{2}$ Drug solubility has a significant impact on bioavailability, therefore selecting and optimising an appropriate drug formulation is imperative to the success of the program; moreover there is currently a very high prevalence of low solubility drugs in development $(70 \%)^{3}$. Although solubility can be estimated through in vitro dissolution studies, and performance predicted through insilico modelling tools, the full impact can only be truly assessed once the drug has been administered to humans in a clinical trial ${ }^{4}$. Although formulation technologies are available that can help to reduce the impact of solubility challenges on bioavailability. Formulation approaches are broadly classified into two categories: drug substance modification and drug product (formulation) modification. In modifying the drug substance, the chemical form of the drug can be changed by generating a new polymorph or salt, and the physical form of the drug can be amended by reducing the 
particle size or crystallinity. In each case, the dissolution rate and/or solubility of the drug may be enhanced ${ }^{5,6}$. However, if these approaches prove unsuccessful, formulations can also be designed to improve solubility. Examples of these include cyclodextrin complexes, lipid-based formulations, suspensions, nanoparticles, nanosuspensions, and spray dried dispersions; all of which contain solubility-enhancing excipients appropriate for the particular drug substance. Once the drug substance or formulations have been modified, it must be evaluated to see whether the solubility has improved $^{7}$.

Nebivolol hydrochloride is a BCS class II drug and receptor blocker with nitric oxide-potentiating vasodilator effect. It is used in treatment of hypertension and is highly cardioselective. It lowers blood pressure by reducing peripheral vascular resistance and significantly increases stroke volume with preservation of cardiac output. Nebivolol has reduced typical beta-blocker related side effects such as fatigue, clinical depression, bradycardia, and impotence. Nebivolol has half-life of about $10 \mathrm{hrs}$. It reaches mean peak plasma concentration approximately in 1.5 to $4 \mathrm{~h}$ post oral administrations ${ }^{8}$. In such cases it is very essential to enhance onset of action of a drug. Therefore, in this work the goal has been set to design, development and characterization of a mucoadhesive controlled-release nanoperticles of Nebivolol using biodegradable polymer Chitosan.

\section{MATERIALS AND METHODS}

\section{Materials}

Chitosan (CS) was obtained as gift sample from Central Institute of Fisheries Technology (Cochin, India). Sodium tripolyphosphate (TPP) was procured from Loba Chemie Pvt. Ltd. (Mumbai, India). Nebivolol (NEB) was obtained as a gift sample from Lupin Ltd. (Pitahmpur, Indore, India). All other chemicals and reagents were of analytical grade.

\section{Preparation of NPs}

The NB-loaded NPs were fabricated according to the procedure reported by (Ajun et al. 2009, Patel et al. 2013) ${ }^{9}$. Briefly, Chitosan solutions of different concentrations were prepared by dissolving chitosan in $1 \%$ aqueous acetic acid solution. Tween $80(2 \% \mathrm{v} / \mathrm{v})$ was added as a surfactant to it under constant stirring at room temperature. Subsequently, drug $(2.5 \%)$ was dissolved in dichloromethane $(2.5 \mathrm{~mL})$, and then this oil phase was added dropwise to the aqueous phase. This addition was accompanied by continuous stirring for 5 minutes at different speeds using high speed homogenizer. Finally, 10ml TPP solution of different concentration was added drop wise into o/w emulsion to induce cross-linking of the particles under magnetic stirring at $500 \mathrm{rpm}$. The stirring was continued to ensure complete evaporation of dichloromethane, it was kept overnight at $40^{\circ} \mathrm{C}$. Nanoparticles were collected by centrifugation at $15,000 \mathrm{rpm}$ for 25 minutes at $20^{\circ} \mathrm{C}$ using cooling centrifuge. The supernatant was subjected for the determination of presence of free Nebivolol by UV spectrophotometer (UV 1700, Shimadzu, Japan).

\section{Characterization of NPs}

\section{Transmission electron microscopy (TEM)}

The morphology of nanoparticles was observed under transmission electron microscopy (Morgagni 268D TEM instrument, AIIMS, New Delhi).

\section{Scanning electron microscopy (SEM)}

The particles were characterized by Scanning Electron Microscopy (SEM, Jeol JSM-6360LV) at a voltage of $20 \mathrm{kV}$ after prior coating with gold/palladium under vacuum by sputtering using a BAL-TEC apparatus.

\section{Drug-excipient compatibility studies by differential scanning calorimeter (DSC)}

The nanoparticles and drug powder were subjected to previously calibrated differential scanning calorimeter (DSC-60, Shimadzu Corporation, Japan). The sample was sealed hermetically in an aluminum pan and subjected to nitrogen gas at a flow rate of $50 \mathrm{ml} / \mathrm{min}$. The thermograms were obtained at scanning temperature range of $50-250^{\circ} \mathrm{C}$ at a heating rate of $10^{\circ} \mathrm{C} / \mathrm{min}$. DSC thermograms were recorded for $\mathrm{CS}, \mathrm{NEB}$ and NEB-CS NPs.

Measurement of particle size, polydispersity index (PDI), and zeta potential (ZP) of NPs

Particle size, PDI and ZP of nanoparticles were determined through Dynamic light scattering (DLS) analysis with Malvern Zetasizer Nano S (Malvern, UK). The analysis was performed in triplicate at a temperature of $25^{\circ} \mathrm{C}$.

\section{Determination of entrapment efficiency}

The entrapment efficiency of the nanoparticulate formulation was determined in triplicate using ultraviolet spectrophotometer. The nanoparticles were separated from the aqueous medium (containing unentrapped NEB) by centrifugation at $25000 \mathrm{rpm}$ for 30 min (REMI CPR-24 Plus, Remi Elektrotechnick, India). The supernatant was diluted with an appropriate amount of $0.1 \mathrm{~N} \mathrm{HCl}$ and analyzed for the amount of unentrapped drug by UV-Visible spectrophotometer (Shimadzu 1700, Japan) at $285 \mathrm{~nm}$.

The percentage drug encapsulated was determined by following the formula:

Entrapment efficiency $(\%)=\frac{\text { Total drug }(\mathrm{mg})-\text { free drug }(\mathrm{mg})}{\text { Total drug }(\mathrm{mg})} \times 100$

\section{In vitro drug release studies}

The in-vitro drug release of nanoparticles was studied by using dialysis membrane (Himedia, India) with a pore size of $2.4 \mathrm{~nm}$ and molecular weight cut-off between 12,000-14,000 in phosphate buffer saline (PBS) pH 7.4 at $37 \pm 2{ }^{\circ} \mathrm{C}$. The amount of drug released was analyzed spectrophotometrically at $285 \mathrm{~nm}$ for NEB

\section{Accelerated stability studies}

Nebivolol loaded nanoparticles were subjected to a stability testing for three months as per International Conference on Harmonisation (ICH) Q1A guidelines. 
Freshly prepared nanoparticles were transferred to $5 \mathrm{ml}$ glass vials sealed with plastic caps and were kept in stability chamber (Remi SC-12 Plus, Remi Instruments. Ltd. Mumbai, India) maintained at $25 \pm 2^{\circ} \mathrm{C} / 60 \pm 5 \% \mathrm{RH}$ for a period of total 3 months. The formulations were monitored for changes in particle size, zeta potential and entrapment efficiency.

\section{RESULTS AND DISCUSSION}

Particle size analysis by transmission electron microscopy (TEM)

The structural morphology of nanoparticles was examined by TEM. TEM image showed that the optimized formulation is nearly spherical in shape and a smooth surface distributed throughout the sample (Figure 1).

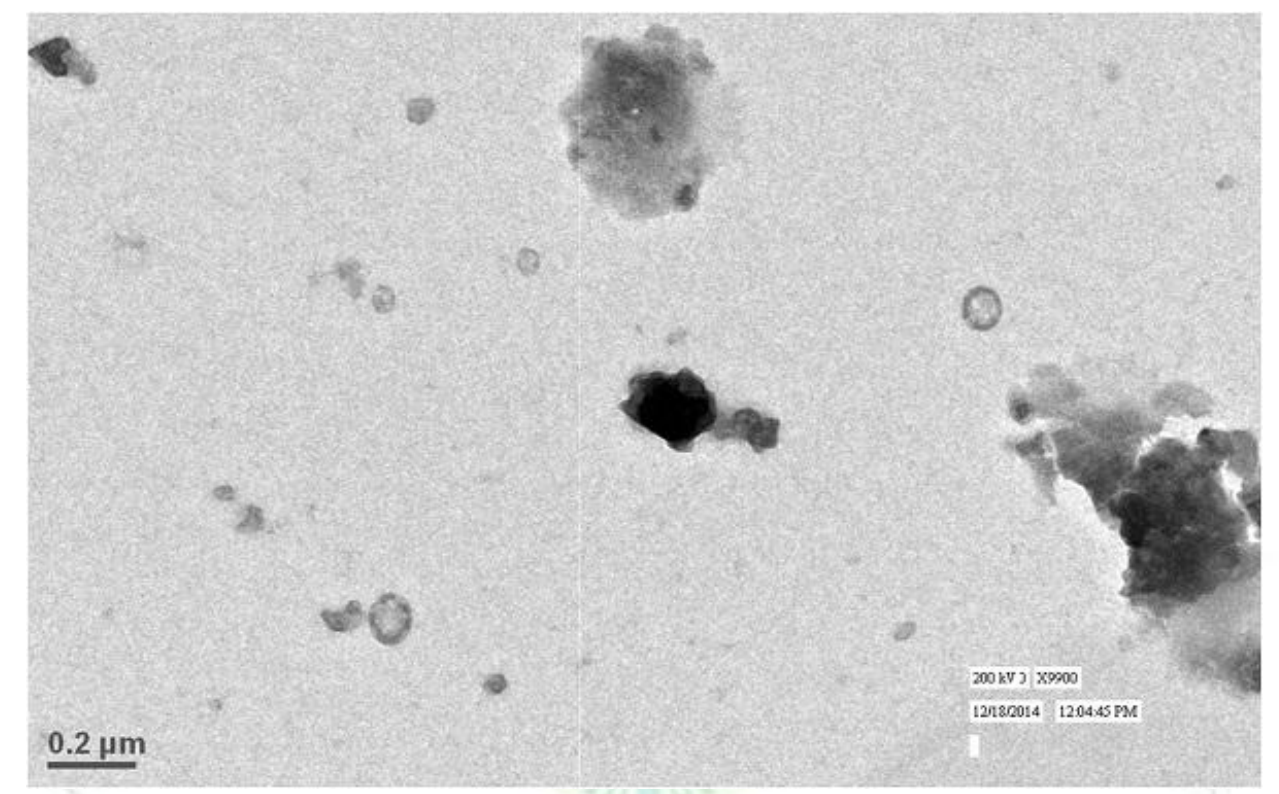

Figure 1: TEM image of the nanoparticles

\section{Particle Morphology analysis by Scanning electron microscopy (SEM)}

The SEM images of the nanoparticles showed spherical, smooth and homogeneous particles (Figure 2).

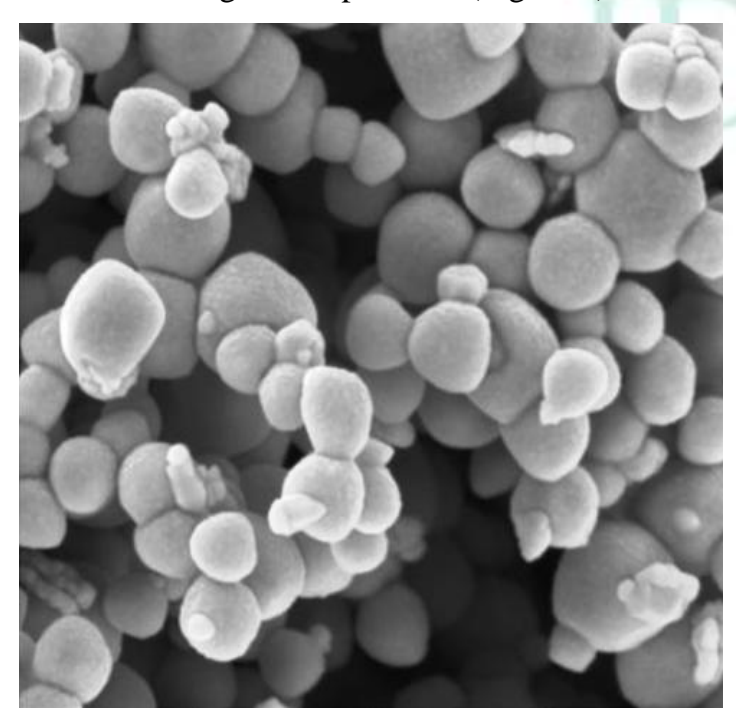

Figure 2: SEM image of the nanoparticles

\section{Drug-excipients compatibility studies by DSC}

The DSC curves of CS, NEB and drug loaded CS NPs were obtained. There is no detectable endotherm if the drug is present in a molecular dispersion or solid solution state in the polymeric nanoparticles ${ }^{10}$. It was seen that the DSC thermograms of drug loaded CS NPs showed a broad endothermic peak at $101.66^{\circ} \mathrm{C}$ which was due to the glass transition temperature of chitosan. However, the melting peak of drug was absent in the thermogram of NPs indicating that NEB was incorporated in amorphous form in the CS matrix in the NPs.

\section{Particle size, poly dispersity index (PDI) and zeta potential of nanoparticles}

The average particle size of the nanoparticles was found to be $79.23 \pm 45 \mathrm{~nm}$ (Figure 3 ). Particle size along with zeta potential $(\zeta)$ is the critical factor that affects the biological performance of chitosan nanoparticles. The zeta potential of NB-CS-NPs were found to be + $36.4 \mathrm{mV} \pm 2 \mathrm{mV}$ (Figure 4 ), which indicate the physical stability of the formulation. The zeta potential also tends to affect particle stability and mucoadhesivity. 


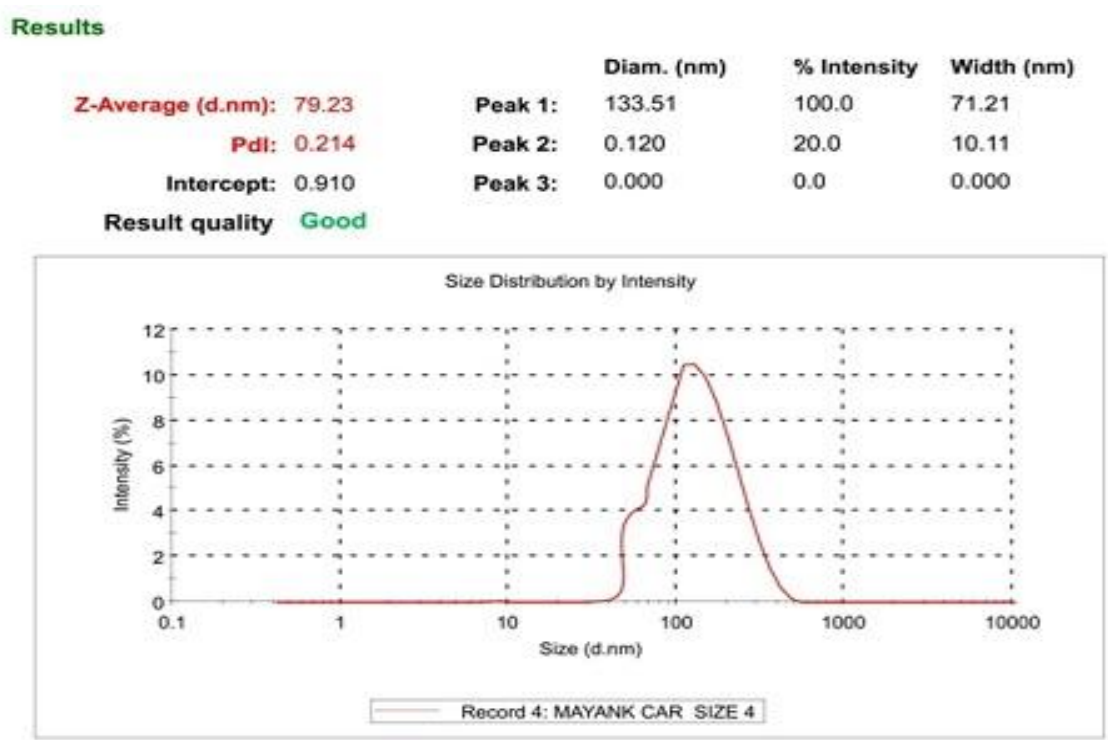

Figure 3: Particle size of the nanoparticles

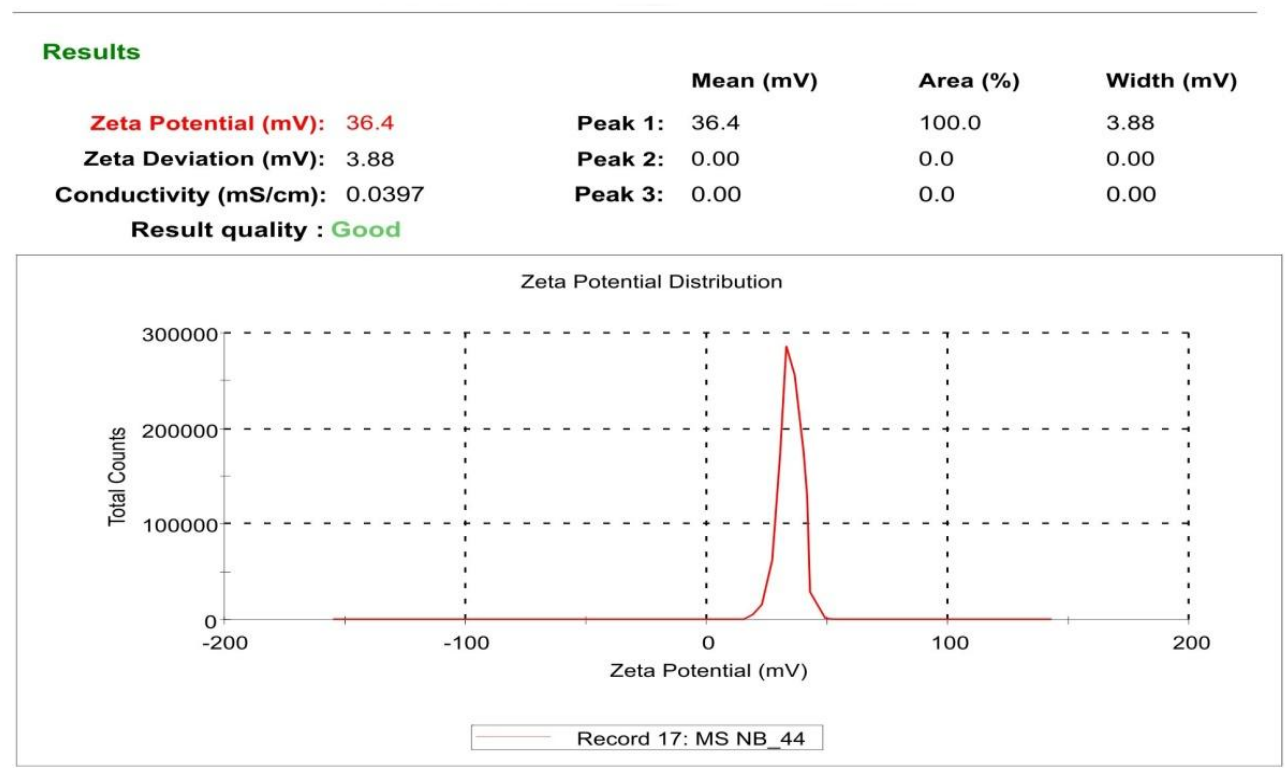

Figure 4: Zeta potential of nanoparticles

\section{Entrapment efficiency}

The entrapment efficiency acts as an important factor influencing the drug release, as well as the overall efficacy of the formulation. All the formulations were analyzed for entrapment efficiency by using UV-Visible spectrophotometer (Shimadzu 1700, Japan) at $285 \mathrm{~nm}$ and. The entrapment efficiency of the nanoparticles was found to be $72.56 \%$.

\section{In vitro drug release}

The In vitro drug release studies were carried out for NEB -CS NPs and marketed formulation in PBS 7.4 at $37{ }^{\circ} \mathrm{C} \pm 2{ }^{\circ} \mathrm{C}$. The drug release profile of NB-CS-NPs showed biphasic release pattern with an initial burst release in the first $2 \mathrm{~h}$ followed by a controlled release over a period of 72 hours and cumulative percentage of drug released was obtained to be $71.24 \%$. (Figure 5)

\section{Accelerated stability studies}

Stability studies were conducted in triplicate for optimized formulation which showed slight variations in particle size, zeta potential, and drug entrapment during 3 months of storage. The obtained results indicated no significant change in the particle size, zeta potential, and drug entrapment during 3 months of storage that ensured the stability of nanoparticles.

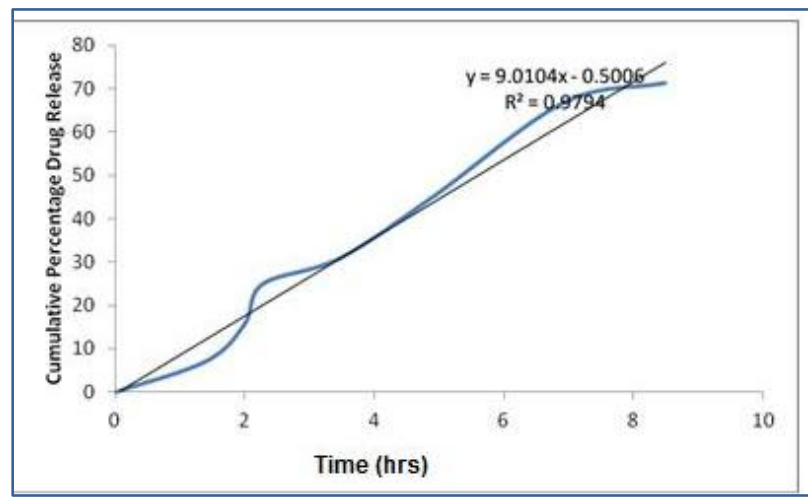

Figure 5: Cumulative percentage of drug released from nanoparticles 


\section{CONCLUSION}

The major challenge in the formulation development is the poor aqueous solubility of the new chemical entity or existing drug molecules. The formulation of these molecules by the application of conventional approaches is difficult and associated with several pharmacological or therapeutical performance issues. The nanoparticles provide a promising approach for enhancing solubility

\section{REFERENCES}

1. Whelton P, He J., Appel L. J. Primary prevention of hypertension: Clinical and public health advisory from the National high blood pressure education programme. JAMA. 2002; 288(15):1882-1888.

2. Neduri K, Bontha V.K, Vemula SK, Different techniques to enhance the dissolution rate of lovastatin: Formulation and evaluation. Asian Journal of Pharmaceutical and Clinical Research, 2013; 6:56-60.

3. Hauss D, Oral lipid-based formulations, Advanced Drug Delivery Reviews, 2007; 59:557-676.

4. Grass GM, Sinko PJ, Effect of diverse datasets on the predictive capability of ADME model in drug discovery, Drug Discovery Today, 2001; 6(12):S54-S61.

5. Kawashima Y. Nanoparticulate systems for improved drug delivery, Advanced Drug Delivery Reviews, 2001; 47:1-2. and oral bioavailability of water insoluble drugs. In conclusion, formulation of chitosan nanoparticles could be an effective strategy for enhancing oral bioavailability of nebivolol and other lipophilic drugs upon further in vivo pharmacokinetics and pharmacodynamics studies.

\section{Conflict of interest: None}

6. Musther $H$. et al., Animal versus human oral drug bioavailability: do they correlate? European Journal of Pharmaceutical Sciences, 2014; 57:280-291

7. Abrahammson B. et al., Extrapolation of Preclinical Data to Predict Human Pharmacokinetics: Understanding and Practice, AAPS Annual Meeting (Los Angeles, California, 2009).

8. Na Z, Larry LA, The influence of swelling capacity of super disintegrate in different $\mathrm{pH}$ media on the dissolution of hydrochlorothiazide from directly compressed tablets.,AAPS Pharm.Sci.Tech., 2005; 6(4):E120-E126.

9. Ajun W, Yan S, Li G, Huili L, Preparation of aspirin and probucol in combination loaded chitosan nanoparticles and in vitro release study. Carbohydrate Polymers. 2009; 75(4):566574.

10. Dubernet $\mathrm{C}$, Thermoanalysis of microspheres, Thermochim Acta, 1995; 248:259-269. 